\title{
El reto de crear bienes culturales en Costa Rica: la producción de libros por parte de las editoriales literarias independientes
}

\author{
Priscilla Carballo-Villagra* \\ (iD) https://orcid.org/0000-0002-7328-0352
}

\author{
Recibido: 04 de octubre, 2018 - Aceptado: 13 de diciembre, 2018
}

\begin{abstract}
RESUMEN
En el presente artículo se exponen los principales resultados de investigación de un proyecto realizado durante el 2017 con editoriales independientes literarias en Costa Rica. Se analizan las características que tiene el proceso de producción de libros en el país por parte de estas editoriales, a la vez que se determinan las principales limitaciones a las que se enfrenta el sector editorial independiente.
\end{abstract}

Palabras clave: Literatura, editoriales independientes, cultura, economía naranja.

El presente artículo es parte de los resultados de un proceso de investigación desarrollado como investigadora independiente con el apoyo del Colegio de Costa Rica del Ministerio de Cultura de Costa Rica; entidad que, por medio de una beca, hizo posible realizar la investigación «El circuito editorial independiente en Costa Rica: Un estudio de la dinámica de producción y comercialización de los sellos editoriales literarios durante el período 2016-2017». Los resultados que se presentan refieren a uno de los dos

\section{Formato de citación según APA}

Carballo Villagra, P. (2019). El reto de crear bienes culturales en Costa Rica: la producción de libros por parte de las editoriales literarias independientes. Revista Espiga, 18 (37), 40-51.

\section{Formato de citación según Chicago-Deusto}

Carballo Villagra, Priscilla (2019). «El reto de crear bienes culturales en Costa Rica: la producción de libros por parte de las editoriales literarias independientes». Revista Espiga 18, n. ${ }^{\circ} 37$ (enero-junio, 2019): 40-51.

Máster en sociología de la Universidad de Costa Rica (UCR) y doctoranda en Estudios de la Sociedad y la Cultura de la UCR. Consultora independiente en temas de género, cultura y políticas públicas e investigadora del Centro de Investigación en Cultura y Desarrollo de la Universidad Estatal a Distancia, de Costa Rica. Correo: pcarballo@uned.ac.cr 
componentes en cuestión, que es la producción de libros por parte de estas editoriales literarias independientes durante el período de estudio.

El trabajo de campo consistió en un mapeo de estas editoriales y un total de 12 entrevistas semiestructuradas, con los siguientes criterios de selección: que fueran editoriales independientes (aspecto que se desarrolla más adelante en el texto), que hubieran publicado libros de literatura de diferentes géneros (poesía, cuento, novela) y que la editorial tuviera al menos un año de existencia y al menos dos libros publicados. A partir de esto las editoriales con las que se trabajó son: Club de Libros, Letra Maya, Uruk, Arlekín, Montemira, Los tres editores, Lanzallamas, La Jirafa y yo, Espiral, Casa Garabato, Edinexo y ZigZag. La autora agradece a cada una por compartir parte de sus experiencias.

\section{Elementos iniciales para el debate}

Para introducir la discusión, se considera fundamental exponer tres elementos para entender el sector editorial independiente en el país.

En primer lugar, es necesario aclarar cómo se entiende el término editorial independiente, para lo que se adscribe los criterios que establece Szpilbarg y Saferstein ${ }^{1}$, a partir de un estudio realizado sobre la industria editorial argentina. Los autores plantean lo siguiente para definir que una editorial sea considerada como independiente:

- Independencia referida a la nacionalidad y tamaño del capital económico, es decir, que sea capital nacional y con bajos márgenes de inversión.

- Independencia referida a la propuesta estética y cultural, es decir, que sea la persona o el equipo de editores quienes deciden los textos a publicar más allá de las líneas editoriales o la posible ganancia.

- Independencia en la organización laboral, al ser empresas pequeñas no existen grandes organigramas, pues incluso en muchos casos son unipersonales o no sobrepasan las cinco personas.

- Independencia en distribución, comercialización y difusión, en donde se utilizan estrategias como redes sociales, blogs, etc.; al no contar con establecimientos propios ni grandes circuitos para esto.

- Independencia en relación con el Estado, en términos de apoyos financieros.

- Independencia de las grandes corporaciones del libro.

Aunque esta independencia es relativa, pues en algunos casos las editoriales utilizan, por ejemplo, algunos apoyos estatales o participan de espacios oficiales, en términos

1. Szpilbarg, Daniela y Ezequiel Saferstein, «El espacio editorial 'independiente': heterogeneidad, posicionamientos y debates: Hacia una tipología de las editoriales en el período 1998-2010» (memoria, Primer Coloquio Argentino de Estudios sobre el Libro y la Edición, Universidad Nacional de la Plata, 2012), acceso: 05 de abril de 2019, http://www.memoria.fahce.unlp.edu.ar/trab_eventos/ev.1955/ev.1955.pdf 
generales se mantienen estas relaciones de independencia tanto con el Estado como con las grandes empresas editoriales o comercializadoras del libro.

En segundo lugar, como un intento de empezar a historizar el proceso de desarrollo de las editoriales independientes, en el trabajo de campo llama la atención que, si se analiza los años de creación de las editoriales, existe un auge del 2000 al 2010, pues en este periodo se crea Arlekín (1999), Casa Garabato (2001), Lanzallamas (2007), Edinexo (2007), La Jirafa y yo (2008) y Espiral (2009). Pero además otras editoriales con las que no se pudo trabajar en esta investigación (pues no mostraron interés en ser entrevistadas), son de este mismo período: Perro Azul (2000), BBB Producciones (2002), Arboleda (2005) y Germinal (2011)2.

A partir de esto, se planteó la pregunta a las personas entrevistadas de por qué consideraban que se dinamizó el sector editorial a partir de este período. Óscar Castillo, director de Uruk, al respecto expone: «Hay una explosión y creo que es porque primero, hay condiciones de apertura del mercado literario. Dos, porque se da mayor conciencia y necesidad de mayor divulgación de bibliodiversidad. Y tres, porque hay una aparición de gentes interesadas y capaces de hacer cosas. A nivel de autores es sorprendente este país $»^{3}$.

De estas razones que aporta Castillo, posiblemente, una de las que generó más eco en relación con los discursos de las otras personas entrevistadas es la necesidad de generar producciones literarias contemporáneas. Sea desde la literatura infantil, como plantearon Casa Garabato y La Jirafa y yo, que empiezan a ver que no existe presencia de historias propias para niños y niñas, o bien, desde la poesía o la narrativa, como explicaron los editores de Lanzallamas ${ }^{4}$ y Espiral ${ }^{5}$. Varias editoriales plantean la necesidad de visibilizar autores contemporáneos.

De esta forma, el surgimiento de editoriales independientes en el país tiene que ver con la necesidad de buscar una voz propia. Como planteó Héctor Gamboa de La Jirafa y yo, en el caso de la literatura infantil y juvenil que publican:

La editorial surge de la idea de la directora de la Escuela Europea, debido a que había una carencia de material de lectura que ofreciera en igualdad de condiciones textos nacionales para escuela. O lo que había era muy antiguo, que es importante por ser parte de la historia, pero que parece que ya pasó y no hay nada más. Este es un tema que tiene que ver con los repertorios y el dinamismo de la cultura. Entonces acá hay naturaleza y problemas que están ausentes en el conocimiento de los niños y crecen pensando que lo que hay es Caperucita o Harry Potter, creen que son las únicas cosas que están presentes. Además, queríamos que hubiera libros accesibles que en términos de calidad compitiera y que los niños no los vean como inferior, más feillos o más chiquitillos ${ }^{6}$.

2. Datos brindados por la Unidad Técnica del Sistema Nacional de Bibliotecas (SINABI), de Costa Rica.

3. Castillo, Óscar, entrevista por Priscilla Carballo, 26 de abril del 2017, oficina de la editorial Uruk, San José.

4. Barquero, Guillermo, entrevista por Priscilla Carballo, 24 de febrero del 2017, cafetería Spoon, San José.

5. Lépiz, Jonathan, entrevista por Priscilla Carballo, 4 de octubre del 2017, cafetería La Cafeoteca, Barrio Escalante, San José.

6. Gamboa, Héctor, entrevista por Priscilla Carballo, 24 de febrero del 2017, oficina de la editorial La Jirafa y yo, Heredia. 
Esto también lo plantean en Lanzallamas desde la narrativa, para quienes desde la academia se habla de la literatura nacional o latinoamericana hacia atrás y se difunde y discute poco de lo actual. Lo anterior da la impresión de que actualmente no se escribe nada más y por esto ellos se proponen reeditar y buscar autores más contemporáneos. Lo cual definitivamente coincide con la discusión sobre el dinamismo en la cultura que expuso Gamboa en la cita anterior.

Al retomar a algunos autores suramericanos, Óscar Castillo denominó esto como «ampliar la bibliodiversidad», es decir, ampliar la diversidad de discursos y referencias bibliográficas más allá de las versiones oficiales de la literatura nacional. Esto, sin duda, es un aporte a la vida cultural del país y es importante tenerlo presente.

En este proceso de posicionar la literatura reciente del país, todas las editoriales entrevistadas plantearon que uno de los aspectos más difíciles es lograr que las personas vieran con igual interés la literatura propia; y, el reto de brindar un producto que pudiera «competir» en calidad narrativa y estética del libro con lo que existe en el mercado venido del extranjero.

Por esto, las personas entrevistadas mencionaron que el camino ha sido complejo, pues han tenido que luchar contra esa idea de las producciones nacionales de inferior calidad, lo cual ha implicado un gran esfuerzo de parte de todas las editoriales por ese proceso de profesionalización. Es decir, las editoriales incluidas en este proceso de investigación son algunas de las que les ha correspondido construir un espacio alternativo de la literatura en ciertos sectores, por lo que es muy relevante conocer el proceso que han seguido y las dificultades que tienen frente a esta circunstancia.

El tercer aspecto fundamental por aclarar al inicio de este análisis, es el hecho de que las editoriales independientes dinamizan un sector de la economía, el de la economía naranja, entendida como la generación de inversiones y ganancias comerciales provenientes de las actividades creativas relacionadas con la cultura. Al respecto:

La economía naranja, como cualquier otro grupo de actividades, se compone de muchos y variados agentes: artistas conceptuales, músicos, escritores, actores, etc. Consumidores, prosumidores, fans, etc. Emprendedores, inversionistas, galeristas, etc. Gestores, críticos, curadores, etc. Empresas, ministerios, agencias, fundaciones, etc. (...) Cada uno de ellos cumple con una o varias funciones que interactúan dentro de un ecosistema muy dinámico, especialmente desde que las tecnologías digitales irrumpieron con todo su ímpetu en la segunda mitad de los años noventa ${ }^{7}$.

Para el caso en desarrollo, este es un elemento que se evidenció a lo largo de todas las entrevistas, pero que posiblemente Ruth Ángulo, de Casa Garabato, sintetizó de la mejor manera: «La industria editorial es como un engranaje de una máquina donde participamos los escritores, los ilustradores, los editores, los diagramadores, los filólogos, el distribuidor, la librería y los promotores de lectura. Es decir, somos muchos $»^{8}$.

7. Buitrago, Felipe e Iván Duque, La economía naranja: una oportunidad infinita (Washington: Banco Interamericano de Desarrollo, 2013), 41.

8. Ángulo, Ruth, entrevista por Priscilla Carballo, 3 de mayo del 2017, oficina de la editorial Casa Garabato, Heredia. 
Este planteamiento es central para empezar a analizar la fase de producción, pues recuerda que el sector editorial dinamiza una serie de pequeños emprendimientos, una serie de microempresas de la cultura a nivel local, ya sean personales, familiares o colectivos, lo que evidencia un aporte importante de este sector al espacio cultural nacional. De esta manera, el apoyo al sector no solo es relevante como posibilidad de escuchar otras voces sobre la realidad costarricense, sino que también tiene un correlato en la realidad económica del país al apoyar pequeñas empresas.

\section{Principales hallazgos sobre el proceso de producción de libros por parte de las editoriales literarias independientes}

En este apartado se presentan los principales elementos que se analizaron de las entrevistas realizadas a las personas de las editoriales en estudio y que permiten ver los retos de producir bienes culturales en la economía costarricense. Para esto, se tomaron en cuenta los siguientes aspectos del proceso de producción:

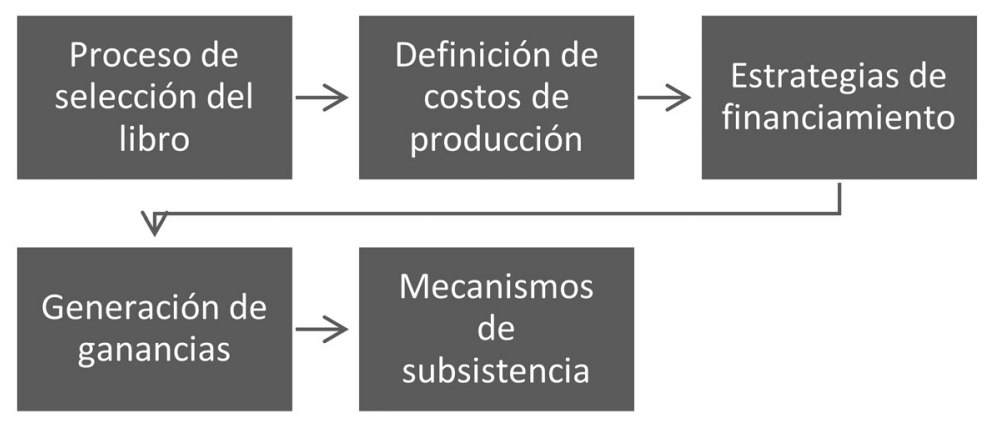

Figura 1. Elementos principales del proceso de producción de libros por parte de las editoriales independientes. Fuente: Elaboración propia.

\section{El proceso de selección de los libros que son parte del catálogo}

En cuanto al proceso de producción, un primer elemento que interesaba conocer es cuál es el proceso para seleccionar un libro para su publicación. La forma de trabajo para recepción de libros de las editoriales es muy variada. Existen editoriales con públicos específicos como Arlekín ${ }^{9}$, que publica trabajos académicos y ensayos, o bien, La Jirafa y yo, que es literatura infantil y juvenil, así como Espiral, que trabaja fundamentalmente poesía. De manera que eso ya funciona como un primer criterio de filtro para decidir si algún texto es viable o no para ser publicado. Además, existen editoriales que reciben de manera abierta manuscritos, mientras que otras, como Los tres editores, plantean que no reciben manuscritos no solicitados y de hecho no los leen, lo que especifican en su página web.

9. Jiménez, Alexánder, entrevista por Priscilla Carballo, 20 de setiembre del 2017, cafetería El Candil, San Pedro de Montes de Oca. 
Luego de esto viene la función del consejo editorial, que depende de la conformación de la editorial, pues en algunas es solo una persona la dueña de la editorial, en otras son dos personas y en otras hasta seis. En todos los casos, estas personas se encargan de leer el manuscrito y de emitir un criterio de la calidad y nivel de madurez del libro.

En editoriales más grandes, como Uruk, que tiene una cantidad de títulos y tirajes significativa, trabaja con lectores externos a quienes se les solicita revisar una obra y dar su criterio; pues, por el volumen de libros, en ocasiones es imposible leer cada manuscrito, por lo que se lo delegan a personas que tienen reconocido criterio literario para que emitan un dictamen. Una vez realizado esto, de acuerdo con la dinámica de cada una de las editoriales, se toma la decisión de aprobar o rechazar el manuscrito.

En algunas de las editoriales el proceso no finaliza ahí, pues si se considera que el manuscrito no es publicable como está, pero que tiene potencial, se le brinda acompañamiento o asesoría al escritor o escritora para mejorar el texto, como lo hace Letra Maya $^{10}$. De esta forma, el trabajo de corrección y madurez de la idea, de los personajes, de las estrategias narrativas, solo se realiza en algunas de las editoriales, debido al tiempo y costo que este trabajo implica; en otros casos, no se hace este trabajo, ya que el autor presenta resistencia a las críticas de sus obras.

En el caso de Edinexo ${ }^{11}$, que trabaja bajo el esquema de autopublicación, no existe un proceso de filtro, sino que vende servicios editoriales al autor: revisión filológica, diagramación, diseño de portada, etc., por lo que no se da una labor de selección. Edinexo denomina esta forma de publicación como «autopublicación asistida». Para el momento de la entrevista, esta editorial indicó que se encontraba desarrollando tres nuevas líneas: ensayo, narrativa y poesía, en las que sí estaba conformando un equipo que le brinde dictámenes que sirvan de filtro de ingreso, pero ese proceso es reciente.

Los criterios que median en el proceso de aceptación de un libro son tan variados como las editoriales mismas, pues no se planteó con demasiada claridad cuáles son estos. Se aplican elementos subjetivos como que el texto le guste al dueño de la editorial, que no sea literatura comercial, que no sea una forma de narración plana y descriptiva o que tenga un objetivo comunicativo claro, entre otros. De manera que no son criterios unificables y cada editorial define sus propios parámetros.

El criterio de que una obra tuviera potencial de ventas se mencionó en las entrevistas realizadas como un elemento por considerar; mas no como algo determinante, pues varias editoriales plantearon que una obra muy vendible al mismo tiempo puede «financiar» la posibilidad de publicar otra no tan rentable; pero por su innovación o discurso contracultural es de interés de la editorial para ser publicada. Este criterio de aporte cultural versus rentabilidad es, sin duda, uno de los elementos culturales más relevantes del sector de editoriales independientes. En un contexto de grandes empresas del libro, donde se tiende a priorizar el best seller, es decir, la venta masiva, el hecho de que estas

10. Fallas, Emilia, entrevista por Priscilla Carballo, 15 de febrero del 2017, cafetería Amandas, Sabanilla de Montes de Oca.

11. Molina, Efrén, entrevista por Priscilla Carballo, 4 de octubre del 2017, oficinas de la editorial, San Pedro de Montes de Oca. 
editoriales pongan más atención al aporte cultural de una obra, aunque no genere muchas ventas, es un elemento importante. Como plantea Danielli:

En ese contexto de concentración -de la edición y de la distribución- y de «supremacía» de criterios de rentabilidad sobre valores culturales, las editoriales independientes -que no forman parte de grupos y donde las decisiones de lo qué editar se toman directamente por sus responsables- cumplen un rol de gran importancia para el desarrollo de las culturas locales y colaboran con la imprescindible diversidad cultural. Son espacios privilegiados donde los escritores e investigadores tienen oportunidad de intercambiar, discutir, reflexionar, allí se inventan en conjunto colecciones, se proponen investigaciones, foros, actuando como agentes culturales, además de publicar esas producciones. (...) El editor independiente es así un factor de diversidad y su actividad refleja el estado de la creación e investigación de una sociedad dada. El editor independiente apasionado compromete sus propios recursos y toma riesgos para publicar una obra innovadora, que tal vez no sea reconocida como tal por el público en el momento de su salida, pero le dará la oportunidad de hacer su camino ${ }^{12}$.

De esta manera es muy estratégico el rol de los editores en tanto agentes culturales, pues son ellos, en la definición del catálogo, quienes definen las líneas de una oferta alternativa en el país.

Un elemento central por destacar en el proceso de selección del catálogo es el mencionado por Los tres editores, que plantea como un criterio publicar literatura escrita por mujeres. Ellos sostienen que su tercer libro va a ser de una mujer y al preguntarles el porqué de ese criterio, Alberto Calvo explica: «Queremos que el tercer libro sea una mujer, porque esa es una tendencia nociva en editoriales, como de seguir la inercia de los que publican más y los que publican más son hombres; entonces, si no hacemos nada, va a seguir siendo así. Siempre nos ha gustado ver qué están haciendo las mujeres, entonces, como editorial, vamos a hacer algo. La realidad es que entre hombres y mujeres hay menos mujeres publicando» ${ }^{13}$.

Esta fue la única editorial que planteó de manera clara ese criterio como un elemento importante de su catálogo, para lo cual se dieron a la tarea de ubicar autoras para empezar un proceso de selección de los trabajos.

\section{Definición de los costos del proceso de producción del libro}

Sobre el proceso de producción del libro, una de las preguntas más difícil de clarificar es la relacionada con el tema de cómo se definían los costos de producción en estas editoriales; no obstante, algunos de los rubros que se mencionaron para definir el valor final de producción de un libro fueron los siguientes:

- Revisión filológica, lo cual se hace solo en algunos casos.

- Asesoría en el desarrollo del contenido del libro que, como se mencionó anteriormente, solo algunas editoriales la realizan. Es decir, varias revisiones del texto, correcciones, recomendaciones de la construcción narrativa, etc.

12. Danielli, Ana, Edición independiente: estrategias para la diversidad (Montevideo: Moneta, C., 2006), 2.

13. Calvo, Alberto, entrevista por Priscilla Carballo, 4 de octubre, San José. 
- El pago por la diagramación del texto y el diseño de la portada, ya que se contrata a una persona de diseño gráfico.

- El pago del proceso creativo de un ilustrador, en el caso de ser necesario de acuerdo con la naturaleza del texto.

- Costo de impresión, es decir, la cantidad de libros que se desea imprimir y la que define la editorial de acuerdo con el potencial que tenga obra. En este apartado entra en consideración el tipo de papel o cartulina, si se usa color para las ilustraciones internas, acabado final de la portada, entre otros.

Ninguna de las editoriales mencionó costos de representación o publicidad para la obra, lo que indica que este rubro no se contempla como una acción específica o necesaria.

Uno de los costos más significativos es el proceso de impresión. En la definición del precio que ofrece una imprenta, existen dos factores relevantes: el tipo de papel y de portada que se utiliza (pues estos materiales son importados y son bastante costosos según su calidad) y la cantidad de pruebas y correcciones que se hacen en la etapa previa de la impresión, pues es en ese momento donde se cuidan muchos de los detalles de la calidad del libro final.

Las editoriales plantearon que, una vez que llegan a una empresa que les satisface en calidad, no cambian, pues prefieren un trabajo según sus expectativas aunque el precio sea, en algunos casos, más elevado. La mayoría trabajaba con imprentas costarricenses, pero una mencionó que trabaja con una imprenta en Estados Unidos y otra ha impreso libros en China, fundamentalmente por el tema de costos y calidad de impresión.

\section{Financiamiento del costo de producción de los libros}

¿Quién paga por la producción de los libros? ¿El autor, la autora o la editorial? En esto se pudo ubicar varias modalidades de distribución de costos, que son las siguientes:

a. Las que le piden al autor que asuma todos los costos, de manera que las editoriales presupuestan y coordinan con todas las partes involucradas (filólogo, dibujante, diagramador). En este caso como parte de su trabajo, la editorial se deja una parte del tiraje final de los libros, lo cual es su ganancia, pues lo distribuyen en librerías o ferias; la otra parte se le da al autor, según la negociación acordada.

b. Las editoriales que asumen todos los costos del proceso, pues se supone que la ganancia está en las ventas. En este caso, se le paga al autor un porcentaje por derechos que normalmente es el $10 \%$ de las ventas, aunque en algunos casos no se les da dinero, sino un porcentaje de los libros impresos equivalente a ese $10 \%$ o al porcentaje acordado.

c. Las editoriales que plantean asumir de manera compartida los costos de producción del libro. En algunos casos cada parte asume el 50\% o varía según la negociación de los porcentajes asumidos.

Estas formas de financiar el proceso dependen de las capacidades financieras de las editoriales, que en general son muy escasas, pues las ganancias de un libro permiten 
tener un pequeño capital de subsistencia, pero no siempre se cuenta con los fondos para financiar una obra. Además, una misma editorial puede trabajar diferentes modalidades de costos, lo que depende de su situación financiera, de manera que en ocasiones puede asumir el costo total de un libro, pero en otro momento es el autor quien asume el costo, dependiendo de la situación. La clasificación de formas de financiamiento no es excluyente ni absoluta.

El volumen de tirajes es variado de acuerdo con la editorial. La mayoría, cuya estructura es pequeña, maneja tirajes de entre 150 y 300 unidades de cada título. Sin embargo, otras que tienen un catálogo y un posicionamiento mayor, hacen tirajes de 1000 a 2000 ejemplares.

En el caso en que la editorial paga todo el proceso, el riesgo evidentemente es mayor y el endeudamiento se vuelve un tema importante. Hay editoriales que corren el riesgo de hacer un préstamo para financiar un libro, pero otras plantean que prefieren tener una cantidad de títulos más pequeña y un crecimiento más lento con tal de no utilizar el crédito como forma de trabajo. De esta manera, la tensa dinámica del financiamiento del libro y del riesgo que implica el endeudamiento, en ciertos casos explica el lento o poco crecimiento de algunas de estas editoriales.

\section{Generación de ganancias en el proceso de producción del libro}

Una vez analizados los factores anteriores de la fase de producción, la gran interrogante es si el resultado genera utilidades. $\mathrm{Al}$ respecto, existen dos posiciones: varias editoriales, generalmente las más pequeñas y con menos títulos, plantean que el porcentaje de ganancia es muy poco, y normalmente lo que se obtiene se reinvierte en el siguiente libro. Las editoriales con catálogos más grandes plantean que sí hay ganancias, aunque no con grandes excedentes, sí para pagar ciertos gastos y el personal.

Un elemento interesante es que la mayoría de las personas de las editoriales no mencionaron cobrar dinero por su trabajo como gestoras de todo el proceso editorial, es decir, no explicitaron un monto por coordinar con filólogos, diagramadores, diseñadores, etc. De manera que gran parte de lo que se recupera se obtiene en la fase de comercialización y ventas, que es donde se obtienen ingresos.

Como se mencionó en apartados anteriores, los costos de los libros varían de acuerdo con el número de páginas, tipo de portada, calidad del papel y el tiraje. Entre más bajo sea el tiraje, más alto es el costo unitario. Por ejemplo, en las ferias del libro a las que se asistió en 2017 y 2018, llamó la atención observar precios de libros de ф5000 o menos; e inevitablemente surge la pregunta de cuál es el monto de ganancia por unidad a cada libro para garantizar la subsistencia de la editorial. Al hacer cálculos para un libro de 150 páginas, la producción oscila entre $₫ 2000$ y $₫ 3000$ la unidad, y si es ilustrado o la calidad de la portada es mayor, posiblemente sube. De manera que si bien se genera ganancia al venderlo en $₫ 5000$ o $₫ 6000$, el principal problema es la recuperación de la inversión en el tiempo, pues los libros pueden quedar almacenados durante meses o años. Y esto se debe contemplar en toda la dinámica editorial. 
Si bien algunas editoriales tienen un manejo de presupuestos y costos como base para fijar sus precios, en varias entrevistas, algunas de las personas se refirieron a que el proceso de fijación del precio de un libro no es muy «científico», en el sentido de que no saben bien cómo establecerlo, pero por lo general se hace de tres formas: uno, se cobra el doble de lo que costó la producción por unidad del libro; dos, se visitan librerías para analizar libros similares en tamaño, calidad y temática para conocer los precios de mercado; tres, se estima la calidad del contenido para fijar el precio de venta al público.

Este proceso de fijación de precios tan poco sistemático es un aspecto que sin duda se debe mejorar en el sector para garantizar su subsistencia. En este tema es importante señalar que la diferencia para la sobrevivencia financiera de una editorial la marca el tamaño del catálogo, pues le permite obtener más ganancia distribuida entre varias opciones de libros y de públicos.

Como se puede observar en el análisis de las editoriales con las que se trabajó, el tema de las estructuras de costos y ganancias es un aspecto de formación pendiente, pues todo se aprende en el camino y, en general, a las personas del sector cultura les cuesta vincularse con estos temas financieros de manera contundente. Este es un aspecto pendiente para todo el sector que vive de la producción cultural; además es relevante para el país, pues garantizaría la subsistencia de circuitos culturales independientes en las diferentes áreas.

\section{Mecanismo de subsistencia financiera de algunas editoriales}

En el proceso de las entrevistas se evidenció que varias de las editoriales diversifican los servicios que brindan para subsistir. En ciertos casos, varios editoriales tienen un emprendimiento paralelo para la venta de servicios editoriales, impresión y encuadernación. En otros, además de la línea de trabajo literario, se venden servicios de edición de libros técnicos o para el sector educativo, ya que la venta de libros para el sector educativo genera más posibilidades de subsistencia, pues existe una población específica y grande para la venta del libro, lo que genera mayor estabilidad.

Otra estrategia es la venta de servicios editoriales para la elaboración de documentos y memorias para el sector empresarial. Finalmente, otra forma de diversificarse es la comercialización de libros de otras editoriales independientes de países como, por ejemplo, de la región centroamericana, de manera que la editorial funciona como distribuidora para Costa Rica.

De esta forma se evidencia la complejidad de la subsistencia de estos proyectos, que obliga a buscar varias formas de financiamiento complementarias para obtener cierto nivel de estabilidad en cuanto a los ingresos.

\section{Reflexiones finales}

Para finalizar este análisis, es importante señalar el papel relevante que tiene la cultura en los países latinoamericanos, como una posibilidad de dinamizar el ideario cultural, político y estético, y en este proceso las editoriales tienen un rol central. Se debe recordar que la creación de relatos implica una lectura de la historia y de la subjetividad de un 
pueblo. La literatura, al igual que la música y cualquier producción cultural, relata una visión de mundo, pero además refleja la disputa para la creación de relatos contraculturales y esto es fundamental en las sociedades latinoamericanas, estructuralmente desiguales.

Por esto, el apoyo a sectores como las editoriales independientes es un asunto de prioridad estatal y social, que de muchas maneras trabajan con serias limitaciones financieras, pese a que existe una dinámica de circulación de libros significativa.

A partir del trabajo de campo realizado, se evidencian varias limitaciones a las que se enfrentan las editoriales independientes; así, sería necesario plantear algunos apoyos para mejorar las condiciones de producción de libros en este sector. A continuación, se plantean las siguientes:

a. No existen políticas de apoyo a las editoriales en temas como abaratamiento de precios en materias primas como papel y tinta, que les ayude a tener costos más accesibles y así enfrentar la fase de producción con mejores condiciones.

b. En cuanto a financiamiento, no hay líneas de crédito particulares para pequeñas empresas del sector cultura. Esto significa que financiar trabajos por la vía del endeudamiento es muy difícil, pues no se les da tratos particulares por sector ni por el aporte a la cultura que realizan. La capacidad de tener «capital semilla» para invertir es muy limitada, por lo que, consecuentemente, el crecimiento del sector es mínimo.

c. Hay mucha incertidumbre en relación con el mercado de lectores, por lo que los tirajes son moderados en cuanto a cantidad y frecuencia en un mercado que lee poco y que no siempre lee títulos de autores nacionales. Por tanto, la falta de estrategias de apoyo a la lectura y de difusión de producción cultural nacional, les afecta considerablemente.

d. Existe un problema de formación de las editoriales en cuanto al cálculo de estructuras de costos, imprevistos, ventas, manejo de inventario, liquidaciones, etc., que se debería solventar. Al tratar de posicionar un producto cultural en un contexto tan pequeño de mercado como el nacional, se debe saber claramente la estructura de ganancias, por lo que es necesario pensar en acciones de capacitación para el sector.

e. Finalmente, una de las limitantes más significativas es que, socialmente, tanto desde el Estado como desde el sector privado, no se ha entendido la relevancia política y cultural de la labor de los editores como agentes culturales. Si esto se analizara con la relevancia que tiene, el apoyo al sector desde diferentes instancias públicas y privadas se podría gestar con mayor facilidad. Justamente, en este elemento de buscar financiamiento alternativo es necesario el vínculo con empresas privadas, por medio de las carteras de responsabilidad social empresarial o el vínculo con organismos financieros internacionales o con organizaciones no gubernamentales, con quienes se ha experimentado poco, por lo que se debe considerar como un espacio interesante.

El sector cultural de las editoriales literarias independientes es un segmento autodidacta en su proceso de aprendizaje, y aún con todas las limitantes, recientemente ha logrado generar catálogos importantes de literatura en el país. Es válido imaginar todo lo que se podría hacer con apoyos específicos desde el Estado y desde otros sectores sociales, por lo que se vuelve fundamental empezar a construir las acciones necesarias. 


\section{ABSTRACT}

\section{The challenge of creating cultural goods in Costa Rica: book production by independent literary publishers}

This article presents the main results from a research carried out during 2017 with independent literary publishing houses in Costa Rica. It proposes an analysis of the production process of books in the country as well as a definition of the main limitations faced by independent publishers.

Key words: Literature, independent publishers, culture, orange economy.

\section{RÉSUMÉ}

\section{Le défi de créer des biens culturels au Costa Rica:} la production des livres par les maisons d'édition littéraires

Cet article expose les principaux résultats d'un projet de recherche effectuée pendant l'année 2017 avec des maisons d'édition indépendantes au Costa Rica. L'article porte aussi sur l'analyse des caractéristiques du processus de production des livres par ces maisons d'édition dans le pays et détermine les principales contraintes auxquelles elles font face.

Mots clés: Littérature, maison d'édition indépendantes, culture, économie orange.

\section{Referencias}

Buitrago, Felipe y Duque, Iván. La economía naranja: una oportunidad infinita. Washington: Banco Interamericano de Desarrollo, 2013.

Calderón, Minor. «La industria editorial y el libro en Costa Rica». Comunicación, n. ${ }^{\circ}$ 1, volumen 18, año 30 (enero-julio 2009).

CERLALC. «Dinámica de la producción editorial centroamericana: un estudio estadístico». Bogotá (2012).

Danielli, Ana. Edición independiente: estrategias para la diversidad. En El jardín de los senderos que se encuentran: Políticas públicas y diversidad cultural en el Mercosur, Moneta, C. (Ed.). Montevideo: UNESCO, 2006.

Espinasa, José María. Las editoriales independientes: una resistencia cultural. 2007. Las editoriales independientes en el mundo latino y la bibliodiversidad. México DF: Fondo de Cultura Económica, 2007.

Fuentes, Lorena y Felipe Castro. La edición independiente en Chile: estudio e historia de la pequeña industria (2009-2014). Santiago: Cooperativa de editores de la Furia, 2015.

Jaramillo, Bernardo. «Ferias, lectura y circulación del libro». En Las ferias del libro: Manual para expositores y visitantes profesionales, Uribe, Richard et al. Centro Regional para el Fomento del libro en América Latina y el Caribe. Bogotá, 2012.

Szpilbarg, Daniela y Ezequiel Saferstein. «El espacio editorial 'independiente': heterogeneidad, posicionamientos y debates: Hacia una tipología de las editoriales en el período 1998-2010». Memoria, Primer Coloquio Argentino de Estudios sobre el Libro y la Edición, Universidad Nacional de la Plata, 2012. Acceso: 05 de abril de 2019. http://www.memoria.fahce.unlp.edu.ar/trab_eventos/ev.1955/ev.1955.pdf 